\title{
The Influence of Sociodemographic Factors on Women's Breast Cancer Screening in Accra, Ghana
}

\author{
Margaret Amenuke-Edusei \\ Charles M. S. Birore
}

\begin{abstract}
Ghana has a relatively low incidence rate of women's breast cancer compared to more developed countries. However, the breast cancer's mortality rate is higher in the former compared to the latter. In Ghana, the role of social work in health care is limited or is not recognized. The purpose of this study was to explore the influence of sociodemographic characteristics, access to healthcare providers, and physicians' recommendations on Ghanaian women's breast cancer screening practices. A crosssectional survey and convenience sample were used to collect data from 194 Ghanaian women after approval was obtained from two Institutional Review Boards, authors of instruments used, and the participants. Univariate, chi-square, and logistic regression statistics were used to analyze data. Seventy-one percent of the participants reported practicing breast self-examination (BSE) and $14 \%$ reported mammogram screening. While educational level and employment were positively associated with BSE, a regular visit to healthcare providers was negatively associated with BSE. Income and physicians' recommendations were positively associated with mammogram screening. Ghanaian women's low level of mammogram screening calls for first, increasing breast cancer awareness and education to counteract negative personal and cultural beliefs relating to breast cancer and screening. Second, social workers in collaboration with health professionals and social justice agencies should advocate and lobby for health insurance legislation which mandates coverage of mammogram screening services. Finally, introducing oncology social work to the curriculum of social work educational programs in Ghana is needed to prepare social workers to address psychosocial challenges relating to breast cancer.
\end{abstract}

Keywords: Breast cancer, breast neoplasm, breast self-examination, mammogram screening, sociodemographic factors, mammography, Africa

Early diagnosis of cancer is critically important because it increases the chances for successful treatment and management of the disease (World Health Organization [WHO], 2019). However, despite advancements in technology and scientific methods in early diagnosis, prevention, and treatment of cancer, women's breast cancer remains a major global health problem, affecting more than 2.5 million women worldwide (Bray et al., 2018; WHO, 2019; 2020). The causes of breast cancer are unknown, but the risk factors include gender, age, previous breast cancer disease, family history of cancer, physical inactivity, obesity, exposure to high radiation, and high levels of stress (Chiriac et al., 2018; WHO, 2018).

\footnotetext{
Margaret Amenuke-Edusei, BA, MPH, PhD, Lecturer and Coordinator of Social Work Program, Department of Social Sciences, Central University, Tema, Ghana, and Charles M. S. Birore, PhD, Associate Professor, The Ethelyn R. Strong School of Social Work, Norfolk State University, Norfolk, VA.
}

Copyright (C) 2020 Authors, Vol. 20 No. 3 (Fall 2020), 756-777, DOI: 10.18060/23663 


\section{Literature Review}

This literature review focuses on a brief overview of the incidence and mortality rates of women's breast cancer in Ghana and other countries, women's breast self-examination (BSE), and mammogram screening. Other variables reviewed include age, marital status, level of education, income, occupation or employment, health insurance coverage, and access to healthcare provider including, regular visits to health care providers and physicians' recommendations for mammogram screening. The three most widely used breast cancer screening procedures across the globe are BSE, clinical breast examination (CBE), and mammography (American Cancer Society [ACS], 2017; International Agency for Research on Cancer (IARC, 2016). Digital mammography is considered the superior method because of its ability to detect extremely small cancerous tumors, which cannot be detected by BSE or CBE (Edgar et al., 2013). In this study, CBE was not investigated because of the limited number of trained healthcare professionals who perform such procedures in Ghana.

\section{Incidence of Breast Cancer}

The latest statistics indicate that in 2018 , the age-standardized incidence and mortality rates of women's breast cancer in Ghana were 34 and 17 per 100,000 women, respectively. In the United States, the age-standardized incidence and mortality rates of women's breast cancer were greater than or equal to 73 and 12.3 per 100,000 women, respectively (Bray et al., 2018; IARC, 2018). Although the incidence rates are higher in more industrialized countries such as the United States than in less industrialized countries such as Ghana, the mortality rates are relatively lower in the former countries compared to the latter. The lower death rates in more industrialized countries are partly attributed to increased women's breast cancer awareness and early detection of the disease. Lack of awareness and screening protocols, limited or lack of access to diagnostic services for early detection, and lower standards of healthcare facilities contribute to higher mortality rates in less industrialized countries (ACS, 2018; da Costa Vieira et al., 2017; Francies et al., 2020). Early diagnosis of breast cancer is important because it leads to early treatment, and thus better outcomes and higher survival rates (ACS, 2018).

Ghana underreports cancer statistics because of inadequate trained personnel and equipment (Laryea et al., 2014). In 2012, the government established the only populationbased cancer registry in Kumasi, the second-largest city in the country. However, the registry is limited to only Kumasi and its immediate surrounding areas. As a result, breast cancer data in the country are largely underreported. A report on cancer statistics in 2014 indicated that breast cancer ranked highest among all the types of female cancer in the registry, representing $36 \%$, followed by cervical cancer $(26 \%)$ and ovarian cancer $(8 \%)$ (Laryea et al., 2014). It is unknown whether the report would have revealed higher statistical rates on breast cancer if the data covered the entire country. Underreported data raises many uncertainties and concerns about the actual incidence of breast cancer among women in Ghana.

Breast self-examination (BSE) involves a woman's self-physical and visual examination of her breasts to determine any unusual changes such as change of skin color, 
detecting a lump, nipple discharge, pain, or size change (ACS, 2017). Currently, BSE is an optional form of breast cancer screening because its effectiveness in reducing mortality rates of women's breast cancer has been questioned (ACS, 2018; NCI, 2017). According to the National Cancer Institute (NCI, 2017), regular BSE is not recommended for breast cancer screening because, in clinical trials, BSE alone does not reduce breast cancer death rates; hence, both ACS $(2018,2019)$ and the United States Preventive Services Task Force (USPSTF, 2016) no longer recommend regular BSE practice. However, Dey (2014) found $\mathrm{BSE}$ to be an important alternative procedure in low-middle income countries where healthcare resources are scarce. Additionally, IARC (2016) noted that women who practiced BSE regularly were more likely to detect breast tumors themselves at their earliest stage and had higher survival rates compared to those who did not.

In Ghana, cultural beliefs play a major role in women's attitudes toward the causes, prevention, and treatment of diseases, including breast cancer (Atobrah, 2012; Opoku et al., 2012). Many Ghanaians attribute diseases to spiritual and supernatural causes. Their strong faith in God, fear of removal of the breasts, and denial are factors associated with reluctance to screen and seek treatment for cancer (Opoku et al., 2012).

Mammogram screening. Mammogram screening involves taking a picture of the breast using $\mathrm{x}$-rays and then looking for tumors and abnormal breast cells in a woman with no symptoms of breast cancer (NCI, 2017). Findings from randomized controlled trials (RCTs) and systematic reviews show that mammograms are effective in detecting breast cancer tumors early, thus, leading to early treatment and reduced mortality rates, especially for those over 50 years (Chan \& So, 2015; Marmot et al., 2013; Myers et al., 2015; Nelson et al., 2016; UK Independent Review Panel, 2012). However, cohort studies have revealed that mammogram screening poses substantial harm because it induces women into overdiagnoses (false-positive test results), which increases the risk of developing cancer (Gøtzsche \& Jørgensen, 2013; Jørgensen et al., 2017). Over-diagnoses expose women to more radiation, stress, breast cancer-specific worry, distress, discomfort, cost of diagnoses, and surgery (Jørgensen \& Gøtzsche, 2009; Nelson et al., 2016). The latest WHO (2020) guidelines for mammogram screening practices no longer recommend such practices among women aged 40-49. While women aged 50-54 should undergo mammography yearly, those aged 55 and older should screen biennially. The latest ACS (2020) guidelines no longer recommend BSE; however, yearly screening should start at age 45-54, and biennial screening should start at age 55 and older. Women have an option of starting mammography at age 40 .

\section{Sociodemographic Factors and Breast Cancer Screening}

A synthesis of the literature shows associations between sociodemographic factors of age, marital status, educational level, income, and employment or occupation, and BSE and mammogram screening (Park et al., 2011). There are mixed findings on the influence of age on breast cancer screening practices. While Tavafian et al. (2009) found a lack of association between age and BSE, Akhigbe and Omuemu (2009) and Park et al. (2011) reported an association between increasing age and BSE. These researchers found participants who practice BSE, or those who undergo mammogram screening are typically 50 years and older. However, other studies have shown that mammography use is more 
common among younger women than older ones and that younger age is positively associated with breast cancer screening practices (Alkhasawneh, 2007; Renshaw et al., 2010; Secginli \& Nahcivan, 2005).

Marital status. A wealth of literature indicates an association between marital status among different racial and ethnic populations and breast cancer screening (Ahmadian et al., 2012; Boxwala et al., 2010; Kiguli-Malwadde et al., 2010). Park et al. (2011) found that among 4,139 Korean women, married women undertook mammography and Papanicolaou smears significantly more than unmarried ones. Similarly, Ahmadian et al. (2012) and Boxwala et al. (2010) reported that more than 65\% of their married participants versus about $20 \%$ of unmarried ones adhered to mammography. Despite the positive correlation between being married and mammogram screening, other researchers (Secginli $\&$ Nahcivan, 2005) found a lack of association between marital status and mammogram screening. In these studies, married women were disproportionately represented, which might have accounted for the differences in the findings. Overall, the literature shows that having a husband, partner, or significant other may either positively or negatively influence breast cancer screening among women across the globe (Hinyard, et al., 2017).

Educational level. Likewise, there are contradictory findings on the influence of educational level on women's breast cancer screening practices. On the one hand, researchers report positive associations (Boxwala et al., 2010; Park et al., 2011) and on the other hand, Esteva et al. (2008) reported a lack of such association. In a sample of 160 Asian-Indian women in the United States, Boxwala et al. (2010) found college-educated women more likely to participate in mammogram screening compared to those without a college education. Opoku et al. (2012) and Renshaw et al. (2010) reported similar findings. By contrast, Esteva et al. (2008) reported that women with a college education had a higher risk of non-attendance and were significantly less likely to participate in a population-based breast cancer-screening program. Additionally, BSE was higher among women without any formal education compared to women who had secondary and tertiary education.

Income. The literature indicates an association between income and breast cancer screening practices (Edgar et al., 2013; Gregory-Mercado et al., 2007; Litaker \& Tomolo, 2007). While Litaker and Tomolo (2007) and Gregory-Mercado et al. (2007) found women from high income households more likely to participate in mammogram screening compared to those from low-income families, Park et al. (2011) and Renshaw et al. (2010) reported an association between living in less affluent or economically deprived areas and non-participation in mammogram screening. These findings underscore the importance of exploring the degree to which income influences women's breast cancer screening practices in Ghana.

Employment and occupation. The literature shows inconsistent associations between breast cancer screening practices and employment status: full-time versus part-time, employed versus unemployed, or retired (Ahmadian et al., 2012; Boxwala et al., 2010; Esteva et al., 2008; Jensen et al., 2012). For instance, Jensen et al. (2012) found that unemployment was associated with non-participation in screening practices. Similarly, Ahmadian et al. (2012) found that among Iranian women, about $67 \%$ of participants who adhered to mammogram screening within the previous two years of the study were 
employed full-time compared to only $16 \%$ of part-time employed or unemployed women. In a sample of 160 Asian-Indian women, Boxwala et al. (2010) reported that almost $75 \%$ of the fully employed participants adhered to breast cancer screening practices compared to their part-time counterparts. In the United States, Litaker and Tomolo (2007) reported similar findings. By contrast, Esteva et al. (2008) reported that more than two-thirds of their participants who had mammogram screening were unemployed or retired compared to about $34 \%$ who were employed.

Access to healthcare providers: The extent to which women in Ghana have access to healthcare providers is not known, although that knowledge is important in the early detection of breast cancer. Recent information on health insurance coverage in Ghana indicated that less than $40 \%$ of the population is covered (Amu et al., 2018); hence, it is necessary to find out how Ghanaian women access healthcare services. Access to healthcare providers in this study comprises health insurance coverage, regular visits to healthcare providers, and physicians' recommendation for breast cancer screening. These variables are reviewed to determine how they influence breast cancer screening.

In many parts of the world, health insurance coverage is reported to have had a strong association with women's breast cancer screening practices (Ahmadian et al., 2012; Sutradhar et al., 2016). The findings of many recent studies indicate that health insurance coverage significantly correlates with participation in breast cancer screening (Ahmadian et al., 2012; Esteva et al., 2008; Park et al., 2011). Thus, this variable was tested among the Ghanaian sample to determine its influence on mammogram screening.

Regular visits to healthcare providers and physicians' recommendations for screening were shown to have positively influenced BSE and mammogram screening practices. Most women learn about breast cancer and the importance of screening through such visits and recommendations create a sense of urgency (Boxwala et al., 2010; Guvenc et al., 2012; Secginli \& Nahcivan, 2005). Guvenc et al. (2012) and Schueler and colleagues (2008) reported that approximately half of their participants received breast cancer and screening information from their physicians and cited physicians' recommendations as a major reason for their mammogram screening.

Using a population-based research approach, the purpose of this study was to explore the influence of sociodemographic characteristics and access to a healthcare provider on women's breast cancer screening practices in Ghana. The broad research questions (RQs) and hypotheses $\left(\mathrm{H}_{0}\right)$ for the study were: RQs: 1. What are the levels of BSE and mammogram screening among Ghanaian women? 2. What are the factors that influence breast cancer screening practices among Ghanaian women? $\mathrm{H}_{1}$ : There are significant levels of BSE and mammogram screening among Ghanaian women. $\mathrm{H}_{2}$ : There are significant associations between sociodemographic factors and access to healthcare provider variables and breast cancer screening among Ghanaian women. 


\section{Methods}

\section{Study Design and Participants}

A cross-sectional survey was used to collect data from a sample of 210 Ghanaian women to explore the influences of sociodemographic characteristics and access to healthcare providers and their recommendations on breast cancer screening. Appropriateness of exploratory and descriptive research, suitability for correlation inquiry, one-point in-time data collection, diverse group sampling, and cost-effectiveness dictated the selection of this design (Creswell \& Creswell, 2018). A study site was randomly selected from eleven Sub-Districts in the Accra Metropolitan Area. The authors selected this area because it includes the capital city of the country and has the few breast cancer screening facilities and trained healthcare professionals in the country.

\section{Ethical Considerations and Procedures}

The Institutional Review Boards (IRB) from a university in the southern United States and the study site country's health service granted permission to conduct this study. The authors completed the online Collaborative Institutional Training Initiative (CITI) program on human subject protection and research integrity. Written permission to use the instruments from the developers was obtained. Participation in the study was announced in the selected churches involved in the study, and informed consent was obtained from all participants. Participants' privacy, confidentiality, and anonymity were maintained.

Sample and sampling. The study involved women aged 18 and older who were residents of the Ayawaso Central sub-District in the Accra Metropolis. This area had approximately 40,000 women (Ghana Statistical Services [GSS], 2012). However, the number of women in the target age group who attended the selected churches in the subDistrict was 1,390. A convenience sample of 210 women without any sign of breast cancer was drawn from two churches. The authors used the Raosoft (2004) sample size calculator to determine the required sample size with 5\% margin of error and $95 \%$ confidence level for this study. Sixteen participants did not complete a substantial number of items in the questionnaire and therefore, were excluded from the data analyses, leaving the final sample size at 194 . The 16 participants constituted less than $10 \%$ of the required sample and thus produced no bias in the results of the study (Langkamp et al., 2010).

\section{Measures}

The authors used a self-administered questionnaire to collect data face-to-face by employing the services of a trained research assistant who distributed the questionnaires to participants and collected them at a later date. The researchers adapted questions from the Breast Health Questionnaire [BHQ] (Shirazi, 2006) to measure breast cancer screening practices and elicited information about participants' sociodemographic characteristics. Regarding sociodemographic characteristics, information was collected on age, marital status, education level, household monthly income, and employment status or occupation. Shirazi (2006) reported the BHQ overall internal consistency reliability ranged from 0.79 to 0.99 and Cronbach's alpha values ranged from 0.69 to 0.88 for its scale and sub-scales, 
respectively. There was no report on Cronbach's alpha for the breast cancer screening items.

The items selected from the BHQ were used for the first time among Ghanaian women. They were suitable to use because they had been tested among women of African descent and the items were clear and easy to understand. The items were also selected based on the literature and recommendations from breast cancer experts. Four items measured BSE and five measured mammogram screening. The Cronbach's Alpha value for the BSE (4-items) in the Ghanaian sample was 0.74. An example of the BSE item was, "Would you say you practiced BSE?" with four response options from $1=$ (once a month), $2=$ (once every 2 months), $3=($ once every 3 months $)$, and $4=($ not at all). Regarding the mammogram screening, participants were asked "Have you ever had a mammogram?" with dummy coded-response options; $1=y e s$, and $0=$ no. The participants who answered "no" to this question responded to an open-ended follow-up question such as, "Can you tell me the reasons why you did not have one?" The participants who answered "yes" were asked "When was your most recent mammogram?" This question had four response options from $1=($ less than one year ago $), 2=(1$ year ago $), 3=($ more than 2 years ago $)$, and $4=($ other $)$. Two yes/no questions asked about participants' personal and family history of breast cancer. These included "Has anyone close to you ever been diagnosed as having breast cancer?" and "Have you ever been told you had breast cancer?" Another question also assessed participants' experiences with breast abnormality with four response options: $1=$ (unusual pain), $2=$ (swelling), $3=($ lump $)$, and $4=($ none of these $)$.

Three yes/no items measured participants' access to healthcare providers (i.e., "Do you have health insurance? Do you have a healthcare provider whom you visit regularly? Has a physician advised you to undergo BSE and/or mammogram screening?)

\section{Data Analysis}

The authors screened the data for accuracy using visual and computerized examinations of entry errors, missing data, and outliers to meet the required assumptions of the selected statistical tests (Tabachnick \& Fidell, 2012). Statistical Package for the Social Sciences (SPSS) version 18.0 was used to analyze data at three levels of statistical tests: univariate using percentages, means, and standard deviations, bivariate using ChiSquare test, and logistic regression.

\section{Results}

\section{Sociodemographic Characteristics}

The sample consisted of 194 women aged 18 to 72 years, with a mean age of 42.15 $(S D=12.80)$. More than half $(53.1 \%)$ of the participants were 40 to 59 years, about $40 \%$ were below age 40 , and $7 \%$ were 60 years and older. A little over half (52.6\%) of the participants were married, $18.6 \%$ were separated, divorced or widowed, and $26.8 \%$ were single or never married. While $42.3 \%$ had completed middle school or some secondary school education, $37.6 \%$ had completed training college. Only $7.2 \%$ of the participants had a university degree, and $2.6 \%$ had no formal education. Regarding type of occupation, 
$26.8 \%$ were traders, $26.8 \%$ were teachers and other civil servants, and $13.9 \%$ were nurses. While $9.3 \%$ identified themselves as self-employed, about $11.3 \%$ reported being unemployed/retired or students.

Table 1. Sociodemographic Characteristics of the Participants $(n=194)$

\begin{tabular}{lr}
\hline Characteristic & $\boldsymbol{n}(\%)$ \\
\hline Age $[M=42.2, S D=12.80]$ & $194(100 \%)$ \\
$18-29$ & $50(25.8 \%)$ \\
$30-39$ & $27(13.9 \%)$ \\
$40-49$ & $103(53.1 \%)$ \\
$60-69$ & $13(6.7 \%)$ \\
$70+$ & $1(0.5 \%)$ \\
\hline Marital Status & \\
Married & $102(52.6 \%)$ \\
Separated/Divorced/Widowed & $36(18.6 \%)$ \\
Never Married & $52(25.9 \%)$ \\
No Response & $4(2.0 \%)$ \\
\hline Level of Education Completed & \\
No Education & $5(2.6 \%)$ \\
Middle/Junior Sec. School & $82(42.3 \%)$ \\
Secondary School & $20(10.3 \%)$ \\
Training College/Diploma & $73(37.6 \%)$ \\
University & $14(7.2 \%)$ \\
\hline Type of Occupation & \\
Nurses & $27(13.9 \%)$ \\
Traders & $52(26.8 \%)$ \\
Teachers/Other Civil Servants & $52(26.8 \%)$ \\
Self-Employed & $18(9.3 \%)$ \\
Unemployed & $3(1.5 \%)$ \\
Retired & $5(2.6 \%)$ \\
Students & $14(7.2 \%)$ \\
No Response & $23(11.9 \%)$ \\
\hline Household Monthly Income in Ghana Cedi \\
Below 500 & $127(65.5 \%)$ \\
500-999 & $26(13.4 \%)$ \\
1,000-2,999 & $21(10.8 \%)$ \\
3,000-4,000 & $15(7.7 \%)$ \\
No Response & $5(2.6 \%)$ \\
\hline Ever Experienced Breast Cancer Signs and Symptoms \\
Unusual Pain, Swelling, Lump & $23(11.9 \%)$ \\
Abnormal Mammogram & $7(3.6 \%)$ \\
No Signs and Symptoms & $160(82.5 \%)$ \\
No Response & $4(2.0 \%)$ \\
\hline Family/Friend with Breast Cancer & \\
Yes & $28(14.4 \%)$ \\
No & $165(85.1 \%)$ \\
No Response & $1(0.5 \%)$ \\
\hline
\end{tabular}


A majority $(65.5 \%)$ of the participants reported a monthly household income below 500 Cedis (approximately $\$ 100$ US in 2020). Regarding family history of breast cancer, $14.4 \%$ reported having a close relative who had breast cancer, and about $12 \%$ experienced unusual pain, swelling, or lump (s) in their breasts. Fewer than $4 \%$ of the participants reported an abnormal mammogram in the previous year. See Table 1 for the complete breakdown of the participants' sociodemographic characteristics.

\section{BSE and Mammogram Screening Practices}

What are the levels of BSE and mammogram screening practices among Ghanaian women?

Regarding the practice of BSE, 192 (99\%) of the 194 participants responded to this question. As presented in Table 2, the majority 136 (70\%) reported that they had practiced BSE. However, only $82(42 \%)$ practiced BSE once a month as recommended by medical experts.

While more than three-quarters $(n=148)$ reported that they knew how to perform BSE, only about half $(n=99)$ reported that a healthcare provider taught them how to perform the procedure.

\begin{tabular}{lr}
\multicolumn{3}{l}{ Table 2. Participants' Number and } \\
Percentage of BSE Practices $(n=194)$ \\
\hline BSE & \multicolumn{1}{c}{ Number (\%) } \\
\hline Ever practiced BSE? & \\
Yes & $136(70.1 \%)$ \\
No & $56(28.9 \%)$ \\
No response & $2(1.0 \%)$ \\
Know how to perform BSE? & \\
Yes & $148(76.3 \%)$ \\
No & $45(23.2 \%)$ \\
No response & $1(0.5 \%)$ \\
Ever taught how to perform $\boldsymbol{B S E} ?$ \\
Yes & $99(51.0 \%)$ \\
No & $94(48.5 \%)$ \\
No response & $1(0.5 \%)$ \\
Frequency of BSE & \\
Once a month & $82(42.3 \%)$ \\
Not once a month & $112(57.7 \%)$ \\
\hline
\end{tabular}

Pertaining to mammogram screening, 117 participants met the eligibility criteria of being 40 years or older to participate in such a screening program. Hence, only this group of the study sample was included in the mammogram screening analysis. As presented in Table 3, $116(99 \%)$ of the participants responded to the question of whether they had ever had a mammogram. Sixteen $(13.7 \%)$ reported they had undergone the screening procedure. Of this number, about half $(n=7,43.75 \%)$ underwent the procedure in the previous year. 
Thirteen $(93 \%)$ of the participants who underwent mammogram screening cited routine regular medical check-up as the reason for their most recent mammogram screening. However, $59(59 \%)$ of the 100 participants who reported non-participation in the screening procedure gave various reasons for not doing so. The most frequent reasons cited were not having signs of breast cancer, not being aware of breast screening, and not receiving recommendations from physicians.

\begin{tabular}{lr} 
Table 3. Participants' Mammogram Screening & Practices $(n=$ \\
\hline \multicolumn{1}{l}{ Mammogram Screening } & $\boldsymbol{n} \mathbf{( \% )}$ \\
\hline Ever had mammogram? & $16(13.7 \%)$ \\
Yes & $100(85.4 \%)$ \\
No & $1(0.9 \%)$ \\
No response & \\
The most recent mammogram & $7(43.75 \%)$ \\
$\leq 1$ year & $7(43.75 \%)$ \\
2 years and more & $2(12.5 \%)$ \\
No response & \\
Reason for the most recent mammogram & $13(92.9 \%)$ \\
Routine medical check-up & $1(7.1 \%)$ \\
Follow-up & $59(59 \%)$ \\
Reason for not having a mammogram & $16(27.1 \%)$ \\
Have no sign of breast cancer & $15(25.4 \%)$ \\
Not aware of screening & $7(12.0 \%)$ \\
Physician did not recommend it & $5(8.5 \%)$ \\
Had not thought about having one & $5(8.5 \%)$ \\
Have financial difficulties & $11(18.5 \%)$ \\
Other reasons &
\end{tabular}

\section{Chi-Square Test Analysis of Sociodemographic Factors}

Are there significant associations between the demographic factors (age, marital status, educational levels, occupation/employment, income) and BSE and mammogram screening among Ghanaian women?

The results of the Pearson's Chi-Square analysis did not show a statistically significant association between age and BSE, $\chi^{2}(1,194)=.81, p=.369$, phi $=.06$. Participants aged 18 to 39 were not different in the practice of BSE from those aged 40 and older. BSE's relative odds ratio value for age $(\mathrm{OR}=1.3)$ showed that older participants were 1.3 times more likely to practice BSE compared to their younger counterparts. In addition, a nonstatistically significant association was found between age category and mammogram screening, $\chi^{2}(1,116)=1.60, p=.281$, phi $=.10$.

The results of the Chi-Square analysis showed a non-statistically significant association between marital status and BSE, $\chi^{2}(2,189)=.85, p=.652$, Cramer's $V=.07$; and marital status and mammogram screening, $\chi^{2}(2,112)=.66, p=.418$, Cramer's $V=$ - 
.077. Additionally, there were no differences in BSE and mammogram screening between married participants and those who were separated, divorced, widowed, or never married.

The Pearson's Chi-Square analysis revealed a statistically significant weak positive association between educational levels and BSE, $\chi^{2}(2,192)=9.09, p=.011$, with a small effect size, indicating Cramer's $V$-value of .22. Participants holding a university education were 3.7 times $(\mathrm{OR}=3.7)$ more likely to practice BSE compared to those with a lower level of education. Participants who completed middle or some secondary education and those who completed college were 2.6 times $(\mathrm{OR}=2.6)$ more likely to practice BSE compared to those who had no formal education or did not complete middle or secondary school, respectively. Non-statistically significant associations were found between educational levels and mammogram screening, Fisher's exact test $(116)=4.64, p=.094$.

The Pearson's Chi-Square and Fisher's exact analyses revealed a statistically significant moderate positive association between occupation/employment status and BSE, $\chi^{2}(3,169)=19.76, p<.001$, with a medium effect size Cramer's $V$-value of .34 (Cohen, 1988). BSE's relative odds ratio showed that nurses were 9.6 times $(\mathrm{OR}=9.6)$ more likely to practice BSE compared to teachers and other workers. BSE's relative odds ratio showed that traders and self-employed participants were four times $(\mathrm{OR}=4)$ less likely to practice BSE compared to teachers and other workers. Non-statistically significant associations were found between occupation/employment and mammogram screening, Fisher's exact test $(108)=3.04, p=.321$.

The results of the Pearson Chi-Square analysis indicated a statistically significant association between household monthly income and mammogram, Fisher's exact test, $(112)=5.14, p=.020$. Participants with monthly household income below 500 Cedis were 4.5 times $(\mathrm{OR}=4.5)$ less likely to participate in mammogram screening compared to their counterparts with household monthly income $\geq 500$ Cedis.

\section{Chi-Square Test Analysis of Access to Healthcare Provider}

Are there significant associations between access to healthcare provider (health insurance coverage, regular visits to healthcare providers, and physicians' recommendations) and BSE and mammogram screening among Ghanaian women?

The results of the Pearson's Chi-Square analysis revealed non-statistically significant associations between health insurance coverage and mammogram screening, $\chi^{2}(1,115)=$ $.102, p=.749$, phi $=-.030)$. Regular visits to the healthcare provider had a weak negative association with BSE, $\chi^{2}(1,191)=5.18, p=.023$, with a small effect size of -.17 phi-value. Participants who did not regularly visit their healthcare provider were 2.2 times $(\mathrm{OR}=2.2)$ less likely to practice BSE than those who regularly visited their healthcare provider. There was no statistically significant association between regular visit to the healthcare provider and mammogram screening, Pearson $\chi^{2}(1,115)=.050, p=.823$, phi $=-.021$.

The Chi-Square result also indicated a statistically significant association between physicians' recommendation and mammogram screening, Fisher's exact test $(115)=11.93$, $p=.001$. Participants who received mammogram screening recommendations from 
physicians were 63 times $(\mathrm{OR}=63)$ more likely to undergo mammogram screening compared to those who did not receive such recommendations.

\section{Logistic Regression Analysis}

Which sociodemographic and access to healthcare provider variables predict BSE and mammogram screening among Ghanaian women?

The results of a binary logistic regression analysis using the forward likelihood-ratio method revealed that occupation, Wald $(1,154)=7.21, p=.007, O R=3.19,95 \% C I(1.37$, $7.50)$, and age $\operatorname{Wald}(1,154)=5.24, p=.022, O R=1.04,95 \% C I(1.01,1.07)$ significantly predicted BSE. Nurses and other civil servants were three times more likely to perform BSE compared to self-employed and unemployed participants. Likewise, older participants were significantly more likely to practice BSE than their younger counterparts.

Physicians' recommendations, Wald $(1,111)=14.01, p<.001, O R=76.60,95 \% C I$ $(8.01,732.57)$ and household monthly income, $\operatorname{Wald}(1,111)=6.93, p=.008, O R=.114$, $95 \% C I(.023, .574)$ emerged as significant predictors. The Cox and Snell $R^{2}$ and the Nagelkerke $R^{2}$ results showed that occupation accounted for $3.1 \%$ to $4.4 \%$, and age accounted for $2.9 \%$ to $4.2 \%$ of the variances in BSE, respectively. The Cox and Snell $R^{2}$ and the Nagelkerke $R^{2}$ results showed that a physicians' recommendation accounted for $22.3 \%$ to $43.4 \%$, and household monthly income accounted for $5.4 \%$ to $10.5 \%$ in mammogram screening, respectively. In total, the model accounted for $27.7 \%$ to $53.9 \%$ of the variance in mammogram screening.

\section{Discussion}

The purpose of this study was to explore the influence of sociodemographic characteristics and access to healthcare provider variables on BSE and mammogram screening practices among women in Ghana. Consistent with the literature (Opoku et al., 2012), this study indicated a moderately high level of BSE and a very low level of mammogram screening practices among the Ghanaian women. Two factors may account for the low level of mammogram screening among the Ghanaian sample. The first one relates to overall negative perceptions about breast cancer and mammogram screening, which Ghanaian women often link to mastectomy or death (Opoku et al., 2012). The second factor relates to the low-income level of approximately two-thirds of the participants.

The findings show that among selected sociodemographic variables, only education and occupation were associated with BSE, and income and physicians' recommendations were associated with mammogram screening. As reported in the literature (Boxwala et al., 2010; Park et al., 2011; Opoku et al., 2012), it appears that women with university or college education are more likely to practice BSE than those with less education. Nurses and teachers were more likely to perform BSE than traders and self-employed or unemployed women. BSE's awareness among nurses and teachers may account for their greater BSE practice compared to other professionals. Such findings underscore the importance of raising BSE awareness through education among women without college or university education. The moderately high knowledge and practice of BSE among more educated women in the sample appear not to have much influence on their mammogram 
screening as indicated in the literature (Akhigbe \& Omuemu, 2009; Ibrahim \& Odusanya, 2009). However, awareness creation alone may be inadequate unless motivation such as pleasure, fear, pain, and prompts are applied as well as developing skills that can influence people's behavior change (Fogg, 2009).

The statistically significant association between higher income and mammogram screening highlights the importance of affordable mammogram screening, which may be a serious concern among the low-income women who cannot afford mammogram screening. Previous studies (Opoku et al., 2012; Park et al., 2011; Renshaw et al., 2010) are consistent with this study's findings and support its hypothesis. Additionally, that finding suggests the need for more advocacy and lobbying by social workers and health-related professionals for national legislation mandating health insurance coverage for mammogram screening services.

Consistent with the works of Parsa et al. (2008) and Tavafian et al. (2009) among Iranian and Malaysian women, non-significant associations were found between age and BSE and mammogram screening. However, other researchers (Akhigbe \& Omuemu, 2009; Park et al., 2011) who studied African and Korean samples observed inconsistencies. For marital status, we found no difference in BSE and mammogram screening between married and unmarried women. Such outcomes were unexpected, but are consistent with previous studies conducted among non-African women (Parsa et al., 2008; Secginli \& Nahcivan, 2005; Tavafian et al., 2009).

As expected, physicians' recommendations were positively associated with mammogram screening; and that observation underscores the critical role of physicians in ensuring mammogram screening among African women. Furthermore, this finding supports the hypothesis and is consistent with the literature (Boxwala et al., 2010; Guvenc et al., 2012; Secginli \& Nahcivan, 2005). Social workers could play an important role in reinforcing the physicians' recommendation for mammogram screening. Working as a team with the physicians and other health professionals in the hospital, clinic, or other health centers, social workers could provide physicians with the outcomes of the psychosocial-emotional and spiritual assessment of the targeted women to help understand their needs and challenges. Despite the strong influence of physicians' recommendation on mammogram screening in this study, it is important to note that only a small percentage of women in this study participated in the procedure. In Africa, including Ghana, there is a high patient-physician ratio. For example, in 2012, there was one physician per 10,000 patients (Abdulai et al., 2017). In 2020, no change was observed (Statista, 2020). Despite this challenge, it is evident that physicians play a major role in encouraging their female patients to undergo breast cancer screening.

In this sample, health insurance coverage and regular visits to healthcare providers were not associated with mammogram screening. We did not expect these findings, as they are inconsistent with the literature (Ahmadian et al., 2012; Esteva et al., 2008; Park et al., 2011). Lack of insurance coverage for mammogram screening poses a threat for many Ghanaian women's ability to screen for breast cancer. The lack of insurance coverage may account for this finding because mammogram screening depends on one's financial 
capability (Opoku et al., 2012). This financial barrier calls for revision of the existing health insurance policy in Ghana that may favor free or subsidized mammograms for women.

About one-third of the participants reported a regular visit to a healthcare provider, and half reported that they had been taught how to perform BSE. However, their regular visits were negatively associated with BSE practice. Additionally, there was no association between regular visits to healthcare providers and mammogram screening. We did not expect these findings because they contradict the literature (Guvenc et al., 2012).

There are four possible explanations for the above findings. First, perhaps there are no breast screening conversations between women and their healthcare providers during these visits. Second, there may be other socioeconomic and cultural factors relating to less emphasis on BSE and mammogram screening on the part of both women and healthcare providers. Third, in Ghanaian society, women rarely undergo mammogram screening because the public considers it unnecessary unless someone has visible signs and symptoms of the illness. Finally, socio-cultural factors contribute to reluctance to undergo mammogram screening including, but not limited to women's fear of cancer diagnosis, death, stigma, and diagnosis procedures; belief in traditional treatment (herbal medicine); and gender roles and household decision-making whereby a woman must consult with a husband or boyfriend for her breast screening (Akuoko et al., 2017). Therefore, it is appropriate to conclude that women's regular visits to healthcare providers do not increase or guarantee BSE and mammogram screening.

The results of the logistic regression analysis suggest that occupation and age predicted BSE, consistent with the literature (Boxwala et al., 2010; Guvenc et al., 2012) and income and physicians' recommendations predicted mammogram screening (Opoku et al., 2012; Park et al., 2011). Nurses and other civil servants among the sample are more likely to perform BSE compared to other participants. As discussed earlier, nurses, teachers, and other civil servants might have received breast cancer and screening information as opposed to other participants. Likewise, older women are more likely to practice BSE compared to their younger counterparts, probably because they are aware that as one grows older the risk of developing breast cancer increases.

\section{Implications of the Findings}

Social workers play varied roles in addressing social and health problems particularly for vulnerable and economically challenged people in society. Such important roles include advocacy, provision of psychosocial services, and linking people to available resources. This study found a moderately high level of BSE and a very low level of mammogram screening practices among Ghanaian women. This information is useful for oncology social workers and other healthcare professionals in Ghana (nurses, public health, medical specialists) practicing in various settings. The first practice implication relates to increased promotion of breast cancer and screening education by social workers, especially oncology social workers and health practitioners. Ghanaian women's low levels of and negative perceptions toward mammogram screening (due to fear of mastectomy and death) should be redressed through health education to mitigate personal and cultural beliefs relating to breast cancer. Oncology social workers can provide psychosocial services, including 
counseling for anxiety and depression associated with screening, diagnosis, and treatment of women's breast cancer. These professionals can work collaboratively with targeted women's groups to educate them about the importance of BSE and mammogram screening for early detection and treatment. Given that oncology social workers do not exist in Ghana, and that there is a limited number of medical oncologists in the country (e.g., only 12 oncology physicians and 59 oncology nurses in 2018) (Matthew, 2018), there is an urgent need for oncology social workers in this country. Introducing oncology social work curriculum in colleges and universities, which offer social work academic degree programs is critical to help address psycho-social-emotional-spiritual problems associated with breast cancer.

The second practice implication involves encouraging women's participation in breast cancer screening practices. It is imperative that women should not only be encouraged to participate in mammography programs, but also should be taught how to perform BSE by paying attention to unusual changes in their breasts in terms of skin color, feeling a painless lump, unusual nipple discharge, or any unusual changes in the breasts. BSE may save many lives by early detection beginning at age 20 and treatment of breast cancer in low-and middle-income countries where health resources and diagnostic tools are insufficient or unavailable (Johnson, 2019). Although ACS (2020) and WHO (2020) no longer recommend BSE, it is still useful for women in resource-constrained countries to detect any abnormality of their breasts early. Depending on assessed risk factors, annual mammogram screening should start at age 40 in such resource-constrained countries (Johnson, 2019).

Our study revealed that educational level and occupation (or employment) was associated with BSE and that income and physicians' recommendations were associated with mammogram screening. These findings have other practice implications for oncology and hospital social workers and other health professionals. First, although education and occupation (employment) are important for improving BSE, they may be inadequate for influencing mammogram screening. Second, while financial resources are critical for mammogram screening, practitioners must consider cultural beliefs toward such screening to improve the screening services, especially in African countries and elsewhere where such beliefs are strong.

The findings showed that physicians' recommendations were positively associated with mammogram screening. However, health insurance coverage and women's regular visits to healthcare providers were not. These findings have further practice implication for oncology and hospital social workers, and health professionals. It appears that when women visit their healthcare providers for breast cancer checkup, and the physicians ask them to undergo the screening procedure, women adhere to their advice. However, women do not normally have conversations with the physicians about cancer, especially breast cancer, and the screening options available if they do not exhibit any such symptoms. Lack of such conversation among women could be associated with fear of mastectomy and its stigma, preference for consulting traditional healers and prayer camps, and lack of or inadequate information about breast cancer and the importance of screening for early detection and treatment. In the future, regionally or nationally representative studies focusing on the influence of cultural values and beliefs, stigma, and fear of breast cancer 
on women's mammogram screening practices would provide useful information for improving such screening practices in Ghana.

Oncology and hospital social workers and other health professionals in Ghana and elsewhere in low- and middle-income countries should deliberately develop breast cancer awareness programs to educate the population and encourage women at risk (based on age and family history of cancer) to have conversations with their healthcare providers about such diseases. The more women are aware of breast cancer, the more knowledge about it, and the more conversations take place with physicians, the more likely that the fear of mastectomy, its stigma, and other socio-cultural beliefs about the disease will be addressed. Given a limited number of medical oncologists in Ghana, it may be important to train all primary care doctors on how to encourage and engage women in conversations regarding breast cancer and the importance of screening practices.

The policy implications of these findings include the development of health insurance policies to cover mammogram services, especially for low-income families. Such health policies at the local, district, and central governments may save many lives in low- and middle-income countries such as Ghana. Social workers and other health professionals in collaboration with social justice agencies locally and internationally have an important advocacy role to play to ensure that policymakers enact appropriate health policies if they are unavailable or are reformed and implemented if they are available. Policy obstacles to improved mammogram screening services in Ghana and other countries like Ghana include lack of government and private employers' investment in employees' health in terms of financing preventive measures such as screening and diagnoses of breast cancer and other diseases. In this regard, social workers are responsible for linking women who need mammogram screening services to available free or subsidized funds for screening and/or treatment that can help ease financial burden on women and their families.

Another policy obstacle relates to the exclusion of funding for mammogram screening by the national health insurance scheme in Ghana. The development and implementation of financial appropriation policy for mammography would ensure high-quality screening outcomes, enhance consistency and high standards of services by healthcare providers, and call for adequate infrastructure in terms of human and financial resources. Excluding women's breast cancer screening and diagnostic services from the national health insurance scheme is a social justice issue. This problem calls for policy advocacy in the sense that social workers and all civil and human rights activists and advocates locally and internationally have ethical and professional responsibilities to tackle such social justice issues. Such exclusion deprives those who need the services and the opportunities to improve the quality of their lives.

\section{Limitations and Future Study}

This study provides information about BSE and breast cancer screening among Ghanaian women. This information may be useful to social workers, health practitioners, other social service professionals, and psychotherapists. However, the study may have limitations worth noting. The first limitation relates to the use of a convenience sample, which limits the generalizability of the findings to the larger population. The second 
limitation concerns the self-reported information, which might have influenced the participants' responses to questions in accordance with what they perceived as socially desirable. Finally, the sample size of 194 may not represent the national state of women's breast cancer screening practices.

Regarding future studies, empirical evidence suggests that increasing awareness and early detection of breast cancer through mammogram screening are the gold standards for effective treatment outcomes and survival. A very low level of mammogram screening in Ghana, therefore, calls for further investigation using a nationally representative sample to determine the extent to which breast cancer knowledge, beliefs, and awareness influence screening practices among Ghanaian women. Such a study would provide useful information to improve prevention strategies, including early detection through screening.

\section{Conclusion}

Ghanaian women's low levels of mammogram screening and negative perceptions toward breast cancer could be addressed through health education to counteract negative personal and cultural beliefs relating to the disease. Although college and university education and occupation or employment appear to be important for improving BSE, they had no influence on mammogram screening in this study's sample. It is important, therefore, to be aware that while financial resources are critical for mammogram screening, practitioners, including oncology and hospital social workers should address cultural obstacles to such screening through health education and counseling to enhance women's participation in mammogram screening, especially in African countries (including Ghana) where such beliefs are strong.

The physicians' recommendation for mammogram screening among African women appears to be an effective strategy for motivating women to undergo breast cancer screening. However, the lack of health insurance coverage for mammogram is a financial barrier for many Ghanaian women. This financial obstacle calls for the public and private health insurance providers to consider including mammogram screening in the national health insurance scheme. Women's regular visits to healthcare providers do not guarantee mammogram screening. The benefits of mammogram screening in low- and middleincome countries such as Ghana, must therefore, be discussed widely by health and social services professionals, and women should be encouraged to perform BSE and engage in mammogram screening.

\section{References}

Abdulai, T., Abobi-Kanbigs, D. A., Joseph, S. K., Adiboka, A. G., \& Solomon, C. (2017). Bridging the inequitable distribution of physicians in Ghana: Factors medical students and house officers at UDS and TTH will consider in accepting postings to northern Ghana. Journal of Healthcare Communications, 2(2), 1-6. https://doi.org/10.4172/2472-1654.100059

Ahmadian, M., Samah, A. A., Redzuan, M., \& Emby, Z. (2012). Predictors of mammography screening among Iranian women attending outpatient clinics in 
Tehran, Iran. Asian Pacific Journal of Cancer Prevention, 13(3), 969-974. https://doi.org/10.7314/apjcp.2012.13.3.969

Akhigbe, A. O., \& Omuemu, V. O. (2009). Knowledge, attitudes and practice of breast cancer screening among female health workers in Nigerian urban city. BioMed Central Cancer, 9(1), 203-312. https://doi.org/10.1186/1471-2407-9-203

Akuoko, C. P., Armah, E., Sarpong, T., Quansah, D. Y., Amankwaa, I., \& Boateng, D. (2017). Barriers to early presentation and diagnosis of breast cancer among African women living in sub-Saharan Africa. PLoS One, 12(2), 1-18. https://doi.org/10.1371/ journal.pone. 0171024

Alkhasawneh, I. M. (2007). Knowledge and practice of breast cancer screening among Jordanian nurses. Oncology Nursing Forum, 34(6), 1211-1217. https://doi.org/ $\underline{10.1188 / 07 . o n f .1211-1217}$

American Cancer Society [ACS]. (2017). Breast cancer: Facts and figures 2017-2018. https://www.cancer.org/content/dam/cancer-org/research/cancer-facts-andstatistics/breast-cancer-facts-and-figures/breast-cancer-facts-and-figures-20172018.pdf

ACS. (2018). The American Cancer Society's principles of oncology: Prevention to survivorship. John Wiley \& Sons. https://onlinelibrary.wiley.com/doi/ book/10.1002/9781119468868

ACS. (2019). American Cancer Society recommendations for the early detection of breast cancer. https://www.cancer.org/cancer/breast-cancer/screening-tests-andearly-detection/american-cancer-society-recommendations-for-the-early-detectionof-breast-cancer.html

ACS. (2020). American Cancer Society breast cancer screening guidelines. https://www.cancer.org/latest-news/special-coverage/american-cancer-society-breastcancer-screening-guidelines.html

Amu, H., Dickson, K. S., Kumi-Kyereme, A., \& Dateh, A. K. M. (2018). Understanding variations in health insurance coverage in Ghana, Kenya, Nigeria, and Tanzania: Evidence from demographic and health surveys. PLoS One, 13(8), 1-14. https://doi.org/10.1371/journal.pone.0201833

Atobrah, D. (2012). When darkness falls at mid-day: Young patients' perceptions and meanings of chronic illness and their implications for medical care. Ghana Medical Journal, 46(2S), 46-53.

Boxwala, F. I., Bridgemohan, A., Griffith, D. M., \& Soliman, A. S. (2010). Factors associated with breast cancer screening in Asian Indian women in Metro-Detroit. Journal of Immigrant and Minority Health, 12(4), 534-543. https://doi.org/10.1007 /s10903-009-9277-0

Bray, F., Ferlay, J., Soerjomataram, I., Siegel, R. L., Torre, L. A., \& Jemal, A. (2018). Global cancer statistics 2018: GLOBOCAN estimates of incidence and mortality 
worldwide for 36 cancers in 185 countries. CA: Cancer Journal for Clinicians, 68(6), 394-424. https://doi.org/10.3322/caac.21492

Chan, D. N. S., \& So, W. K. W. (2015). A systematic review of randomly controlled trials examining the effectiveness of breast and cervical cancer screening interventions for ethnic minority women. European Journal of Oncology Nursing, 19(5), 536-553. https://doi.org/10.1016/j.ejon.2015.02.015

Chiriac, V. F., Baban, A., \& Dumitrascu, D. L. (2018). Psychological stress and breast cancer incidence: A systematic review. Clujul Medical, 91(1), 18-26. https://doi.org/10.15386/cjmed-924

Cohen, J. (1988). Statistical power analysis for the behavioral sciences. Routledge Academic.

Creswell, J. W., \& Creswell, J. D. (2018). Research design: Qualitative, quantitative, and mixed methods approaches (5th ed.). Sage. https://doi.org/10.5539/elt.v12n5p40

da Costa Vieira, R. A., Biller, G., Uemura, G., Ruiz, C. A., \& Curado, M. P. (2017). Breast cancer screening in developing countries. Clinics (Sao Paulo, Brazil), 72(4), 244-253. https://doi.org/10.6061/clinics/2017(04)09

Dey, S. (2014). Preventing breast cancer in LMICs via screening and/or early detection: The real or the surreal. World Journal of Clinical Oncology, 5(3), 509-519. https://doi.org/10.5306/wjco.v5.i3.509

Edgar, L., Glackin, M., Hughes, C., \& Rogers, K. M. (2013). Factors influencing participation in breast cancer screening. British Journal of Nursing, 22(17), 10211026. https://doi.org/10.12968/bjon.2013.22.17.1021

Esteva, M., Ripoll, J., Leiva, A., Sánchez-Contador, C., \& Collado, F. (2008). Determinants of non-attendance to mammogram program in a region with high voluntary health insurance coverage. BioMed Central Public Health, 8(1), 387-396. https://doi.org/10.1186/1471-2458-8-387

Fogg, B. J. (2009). A behavior model for persuasive design. https://endregion.ir/ uploads/weblog/persuasive technology_ref/Fogg\%20Behavior\%20Model.pdf

Francies, F. Z., Hull, R., Khanyile, R., \& Dlamini, D. (2020). Breast cancer in lowmiddle income countries: Abnormality in splicing and lack of targeted treatment options. American Journal of Cancer Research, 10(5), 1568-1591.

Ghana Statistical Service. (2012). The 2010 population and housing census: Summary report of final results. https://www.statsghana.gov.gh/gssmain/storage/img/marquee updater/Census2010_Summary_report_of_final_results.pdf

Gøtzsche, P. C., \& Jørgensen, K. J. (2013). Screening for breast cancer with mammography. Cochrane Database of Systematic Reviews, 2013(6), 1-73. https://doi.org/10.1002/14651858.cd001877.pub5

Gregory-Mercado, K. Y., Will, J. C., True, S., Royalty, J., Starcher II, E. T., Khavjou, O., Helsel, W., Kammerer, W., \& Howe, W. (2007). A combined approach to women's 
health is associated with a greater likelihood of repeat mammography in a population of financially disadvantaged women. Preventing Chronic Disease: Public Health Research, Practice and Policy, 4(4), 1-9.

Guvenc, I., Guvenc, G., Tastan, S., \& Akyuz, A. (2012). Identifying women's knowledge about risk factors of breast cancer and reasons for having mammography. Asian Pacific Journal of Cancer Prevention, 13(8), 1491-1497. https://doi.org/10.7314/ apjcp.2012.13.8.4191

Hinyard, L. J., Wirth, L. S., Clancy J. M., \& Schwartz, T. (2017). The effect of marital status on breast cancer-related outcomes in women under 65: A SEER database analysis. The Breast, 32, 13-17. https://doi.org/10.1016/j.breast.2016.12.008

Ibrahim, N. A., \& Odusanya, O. O. (2009). Knowledge of risk factors, beliefs and practices of female healthcare professionals towards breast cancer in a tertiary institution in Lagos, Nigeria. BioMed Central Cancer, 9(76), 1-8. https://doi.org/10.1186/1471-2407-9-76

International Agency for Research on Cancer [IARC]. (2016). Handbooks of cancer prevention: Breast cancer screening (vol. 15), Lyon, France: IARC Press. https://publications.iarc.fr/Book-And-Report-Series/Iarc-Handbooks-Of-CancerPrevention/Breast-Cancer-Screening-2016

IARC. (2018). Breast cancer factsheet. https://gco.iarc.fr/today/data/factsheets/ cancers/20-Breast-fact-sheet.pdf

Jensen, L. F., Pedersen, A. F., Andersen, B., \& Vedsted, P. (2012). Identifying specific non- attending groups in breast cancer screening: Population-based registry study of participation and socio-demography. BioMed Central Cancer, 12(159), 1-9. https://doi.org/10.1186/1471-2407-12-518

Johnson, O. E. (2019). Awareness and practice of breast self-examination among women in different African countries: A 10-year review of literature. Nigerian Medical Journal, 60(5), 219-225. https://doi.org/10.4103/nmj.nmj_84_19

Jørgensen, K. J., \& Gøtzsche, P. C. (2009). Overdiagnosis of publicly organised mammography screening programmes: Systematic review of incidence trends. British Medical Journal, 339(1), 1-8. https://doi.org/10.1136/bmj.b2587

Jørgensen, K. J., Gøtzsche, P. C., Kalager, M., \& Zahl, P-H. (2017). Breast cancer screening in Denmark: A cohort study of tumour size and overdiagnosis. Annals of Internal Medicine, 166(5), 313-323. https://doi.org/10.7326/m16-0270

Kiguli-Malwadde, E., Mubuuke, A. G., Businge, F., Kawooya, G. M., Nakatudde, R., Byanyima, K. R., \& Muyinda, Z. (2010). Current knowledge, attitudes and practices of women on breast cancer and mammogram at Mulago Hospital. Pan African Medical Journal, 5(1), 9-21.

Langkemp, D. L., Lehman, A., \& Lemeshow, S. (2010). Techniques for handling missing data in secondary analyses of large surveys. Academic Pediatrics, 10(3), 205-210. https://doi.org/10.1016/j.acap.2010.01.005 
Laryea, D. O., Awuah, B., Amoako, Y. A., Osei-Bonsu, E., Dogbe, J., Larcen-Reindorf, R., Ansong, D., Yeboah-Awudzi, K., Oppong, J. K., Konney, T. O., Boadu, K. O., Nguah, S. B., Titiloye, N. A., Frimpong, N. O., Awittor, F. K., \& Martin, I. K. (2014). Cancer incidence in Ghana, 2012: Evidence from a population- based cancer registry. BioMed Central Cancer, 14, 362-370. https://doi.org/10.1186/1471-2407-14-362

Litaker, D., \& Tomolo, A. (2007). Association of contextual factors and breast cancer screening: Finding new targets to promote early detection. Journal of Women's Health, 16(1), 36-45. https://doi.org/10.1089/jwh.2006.0090

Marmot, M. G., Altman, D. G., Cameron, D. A., Dewar, J. A., Thompson, S. G., \& Wilcox, M. (2013). The benefits and harms of breast cancer screening: An independent review. British Journal of Cancer, 108(11), 2205-2240. https://doi.org/10.1038/bjc.2013.177

Matthew, A. (2018). Global survey of clinical oncology workforce. Journal of Global Oncology, 4, 1-12. https://ascopubs.org/doi/pdfdirect/10.1200/JGO.17.00188

Myers, E. R., Moorman, P., Gierisch, J. M., Havrilesky, L. J., Grimm, L. J., Ghate, S., Davidson, B., Mongtomery, R. C., Crowley, M. J., McCrory, D. C., Kendrick, A., \& Sanders, G. D. (2015). Benefits and harms of breast cancer screening: A systematic review. Journal of American Medical Association, 314(15), 1615-1634. https://doi.org/10.1001/jama.2015.13183

National Cancer Institute [NCI]. (2017). Mammograms. https://www.cancer.gov/types/ breast/mammograms-fact-sheet

Nelson, H. D., Pappas, M., Cantor, A., Griffin, J., Daeges, M., \& Humphrey, L. (2016). Harms of breast cancer screening: Systematic review to update the 2009 U.S. Preventive Services Task Force Recommendation. Annals of Internal Medicine, 164(4), 257-267. https://doi.org/10.7326/m15-0970

Opoku, S. Y., Benwell, M., \& Yarney, J. (2012). Knowledge, attitudes, beliefs, behavior and breast cancer screening practices in Ghana, West Africa. Pan African Medical Journal, 11, 28-37. https://doi.org/10.1016/s1359-6349(10)70778-3

Park, M. J., Park, E-C., Choi, K. S., Jun, J. K., \& Lee, H-Y. (2011). Socio-demographic gradients in breast and cervical cancer screening in Korea: The Korean National Cancer Screening Survey (KNCSS) 2005-2009. BioMed Central Cancer, 11(257), 18. https://doi.org/10.1186/1471-2407-11-257

Parsa, P., Kandiah, M., Zulkefli, N., \& Rahman, H. (2008). Knowledge and behavior regarding breast cancer screening among female teachers in Selangor, Malaysia. Asian Pacific Journal of Cancer Prevention, 9, 221-227.

Raosoft Incorporated. (2004). Sample size calculator. Seattle, WA. https://doi.org/ $\underline{10.1016 / \mathrm{s} 1470-2045(08) 70175-\mathrm{x}}$

Renshaw, C., Jack, R. H., Dixon, S., Moller, H., \& Davies, E. A. (2010). Estimating attendance for breast cancer screening in ethnic groups in London. BioMed Central Public Health, 10(1), 1-8. https://doi.org/10.1186/1471-2458-10-157 
Schueler, K. M., Chu, P. W., \& Smith-Bindman, R. (2008). Factors associated with mammogram utilization: A systematic quantitative review of the literature. Journal of Women's Health, 17(9), 1477-1498. https://doi.org/10.1089/jwh.2007.0603

Secginli, S., \& Nahcivan, N. O. (2005). Factors associated with breast cancer screening behaviors in a sample of Turkish women: A questionnaire survey. International Journal of Nursing Studies, 43(2), 161-171. https://doi.org/10.1016/j.ijnurstu.2005.02.004

Shirazi, M. A. (2006). Breast cancer screening behaviors among immigrant Iranian women in the United States (Doctoral dissertation). Oregon, Oregon State University.

Statista. (2020). Density of medical doctors in West Africa in 2020 by country (per 10,000 inhabitants). https:/www.statista.com/statistics/1122671/density-of-medicaldoctors-in-west-africa-by-country/

Sutradhar, R., Gu, S., \& Paszar, L. F. (2016). Multi-state transitional models for measuring adherence to breast cancer screening: A population-based longitudinal cohort study with over two million women. Journal of Medical Screening, 24(2), 7582. https://doi.org/10.1177/0969141316654940

Tabachnick, B. G., \& Fidell, L. S. (2012). Using multivariate statistics (6th ed.). Pearson.

Tavafian, S. S., Hasani, L., Aghamolaei, T., Zare, S., \& Gregory, D. (2009). Prediction of breast self-examination in a sample of Iranian women: An application of the Health Belief Model. BioMed Central Women's Health, 9, 37-44. https://doi.org/10.1186/1472-6874-9-37

United Kingdom Independent Panel on Breast Cancer Screening. (2012). The benefits of and harms of breast cancer screening: An independent review. Lancet, 380(9855), 1778-1786. https://doi.org/10.1016/s0140-6736(12)61611-0

United States Preventive Services Task Force. (2016). Final recommendation statement: breast cancer screening. Author. https://www.uspreventiveservicestaskforce.org /uspstf/document/RecommendationStatementFinal/breast-cancer-screening

World Health Organization [WHO]. (2018). Cancer: Key facts. https://www.who.int/en/ news-room/fact-sheets/detail/cancer

WHO. (2019). Cancer: Early diagnosis. https://www.who.int/cancer/prevention/diagnosisscreening/en/

WHO. (2020). Breast cancer. https://www.who.int/cancer/prevention/diagnosisscreening/breast-cancer/en/

Author note: Address correspondence to Margaret Amenuke-Edusei, Central University, Faculty of Arts and Social Sciences, Social Work Unit, P. O. Box 2305, Tema, Ghana. Email: mamenuke@,central.edu.gh 